\title{
Special Issue: NEWCAS 2014 and 2015
}

\author{
Sylvain Bourdel $^{1} \cdot$ Yannis Le Guennec $^{1} \cdot$ Dominique Morche $^{2}$
}

Published online: 1 September 2016

(c) Springer Science+Business Media New York 2016

This special issue of Analog Integrated Circuits and Signal Processing (ALOG) includes the extended version of a selective number of papers that were originally presented at the past editions of the IEEE International NEWCAS conference, held in Grenoble (France) on June 07-10, 2015 and in Trois-Rivières (Québec) on June 22-25, 2014. Since its birth, this IEEE International NEWCAS conference has consistently grown to become a major venue where highly innovative circuit and system design techniques have been presented for the first time. The conference covers a wide variety of subjects including analog and RF circuit design, digital and mixed-signal integrated circuits, computer architecture, signal processing, sensors, encompassing a broad range of applications such as telecommunications, imaging and vision, safety, energy, and biomedical.

In the 2015 NEWCAS edition, the submissions reached the record number of 321 from 45 countries from all over the world. Based on a reviewing process with 3.4 average reviewers per paper, 140 contributions were accepted for presentation with a $43.6 \%$ acceptance rate. This score fits well the general trend of high and yet increasing quality of the conference. This accomplishment is the results of a collective effort supported by

Sylvain Bourdel

sylvain.bourdel@minatec.inpg.fr

Yannis Le Guennec

yannis.leguennec@minatec.inpg.fr

Dominique Morche

dominique.morche@cea.fr

1 IMEP-LAHC, Grenoble - INP (Institut National Polytechnique de Grenoble), 3 Parvis Louis Néel - CS 50257, 38016 Grenoble, France

2 Radio Laboratory, CEA-LETI, 17, Rue des martyrs, 38054 Grenoble, France the Technical Program Chairs, Dr. Shahriar Mirabbasi, Dr. Yannis Le Guennec, and Dr. Sylvain Bourdel, and inspired by the vision of the NEWCAS 2015 General Chairs, Dr. Dominique Morche and Dr. Mohamad Sawan.

Papers of NEWCAS 2014 edition, held in Trois-Rivières (Canada) have also been included in this Special Issue. The conference, chaired by Dr. Daniel Massicotte and Dr. Mohamad Sawan, has been also a great success.

The 12 selected papers included in this special issue address various challenges that are within the scope of NEWCAS, as listed below:

\section{(1) 2 papers}

Analog and RF circuit design: RF and MMW power amplifier design.

\section{(2) 2 papers}

Power management: Analog processing for power networks and voltage references.

\section{(3) 3 papers}

Biomedical: Ultra low power programmable filter, transmitting circuits for CMUTs and resonant converter.

\section{(4) 3 papers}

Microsystems, imaging and sensors: Single-photon avalanche diode, Rogowski coil current transducer, direct light to time converter.

\section{(5) 2 papers}

Digital circuits: Approximate adder synthesis for FIR filters and subsampled adaptive predistortion algorithm.

We would like to thank the authors for their scientific contributions, and the numerous reviewers for their 
constructive evaluations. We would also like to express our appreciation for the continuous and tireless support of the Springer personnel. At last, we are grateful to Professor Mohammed Ismail for giving us the opportunity to lead this special issue.

In the end, this special issue was made possible by the personal contribution of many people. We take the opportunity offered by this Editorial to thank each and all of them.

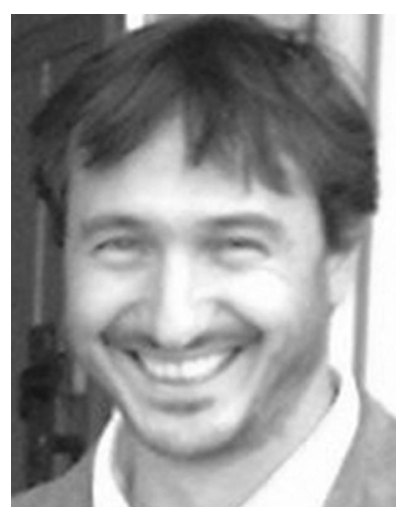

Sylvain Bourdel received the $\mathrm{Ph} . \mathrm{D}$. in microelectronics from the National Institute of Applied Science (INSA) of Toulouse in 2000. He was with the LAAS laboratory of Toulouse where he was involved on radiofrequency systems modelling and he was particularly focused on spread spectrum techniques applied to $2.45 \mathrm{GHz}$ transceivers. In 2002 he joined the IM2NP in Marseille where he headed with $\mathrm{Pr}$ H. Barthélemy the Integrated Circuit Design Team of the IM2NP. He joined in 2013 the Grenoble-INP (Institut National Polytechnique de Grenoble) as a full Professor where he works at the IMEP-LAHC laboratory. He works on RF and MMW IC design and integration. He particularly focuses on low cost and low power applications. His area of interest also includes system level specifications, UWB and RFID. He is the author and co-author of more than 70 referenced IEEE publications.

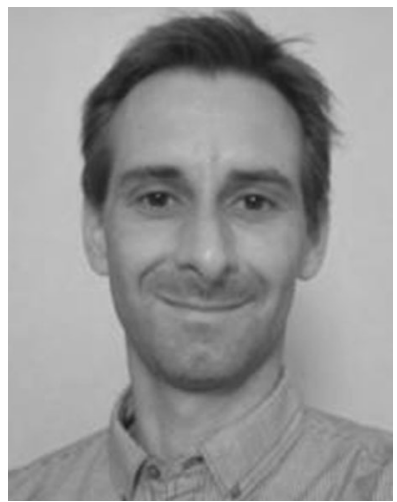

Yannis Le Guennec received the Ph.D degree in Optics Optoelectronics and Microwaves from the Grenoble Institute of Technology in 2003. In 2004, he held a postdoctoral position in National University of Ireland, Maynooth. $\mathrm{He}$ is currently associate Professor at the Institute for Microelectronics, Electromagnetism and Photonics - Laboratory of Hyperfrequencies and Characterization (IMEP-LAHC), Grenoble. His fields of interest are high data rate interconnect systems and related signal processing, all optical signal processing, optical wireless, microwave photonics.

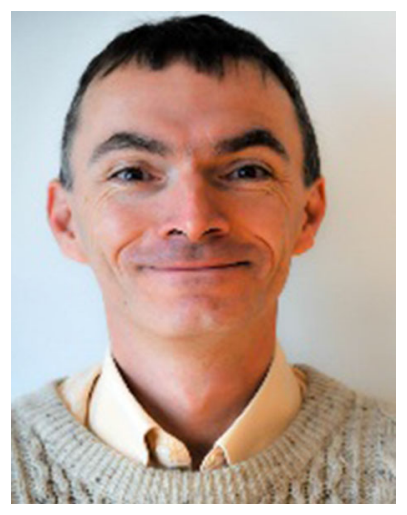

Dominique Morche received the Master of Science (M.S.) degree in engineering from the Ecole Nationale Supérieure d'Electricité et de Radioelectricité de Bordeaux, Bordeaux, France, in 1990 and the Ph.D. degree in electronics from the Institut National Polytechnique de Grenoble, Grenoble, France, in 1994. His Ph.D. mainly focuses on sigma-delta ADCs. From 1994 to 2001, he was with France Telecom, Meylan, France, as a Research Engineer. He has been involved in the architecture and design of analog circuits for telecom applications. He is currently with Commissariat à l'énergie atomique-Leti Minatec, Grenoble, France. His current field of research is in the specification and design of RF architecture for UWB and IoT systems. Since 2013, he is a Research Director of the french Institute and acts a scientific director of the RF labs. So far he has co-authored more than 80 technical publications on journal and international conferences and he holds more than 15 patents. He also serves or has served in the technical comitee of various conference among ESSCIRC, DATE, NEWCAS, ICICDT, ISCDG, PRIME, TAISA, ... He was Technical Program Chair of IEEE NEWCAS 2014 and General Chair of NEWCAS 2015. 